\title{
The use of simple metabolic evaluation in determining the etiology of recurrent stone formers
}

\begin{abstract}
Objectives: Recurrence rates in urolithiasis may reach to 50\% after 5-10 years. Simple metabolic evaluation (SE) is commonly used for evaluating patients with low risk for recurrence. In the present study, patients with high risk to develop for stone recurrence were evaluated by single sample of urine collected in 24 hours urination with standard diet.

Methods: Two hunderd and sixty eight cases who were supposed to be patients with high risk for stone recurrence were evaluated. Fifty three patients who had routine follow up for 5 years were investigated. Calcium, sodium, potassium, magnesium, creatinine, BUN, uric acide, parathormone in blood and calcium, uric acide, magnesium, citrate, urine volume and oxalate in the sample of urine collected in 24 hours urination with standard diet were checked.

Results: Metabolic abnormalities were determined in $87 \%$ of the patients. During the five years, more than 3 parameters corrupted in $9(17 \%)$ and less than three parameters corrupted in $12(23 \%)$ patients. In $5(1 \%)$ patients the same parameters never changed. In $14(26 \%)$ patients all of the parameters improved whereas at least one parameter recovered in $13(25 \%)$ patients. Stone recurrence was seen in $2(4 \%)$ patients.

Conclusion: Metabolic evaluation and preventive medical therapy are supplementary steps in the treatment of urolithiasis. We conclude that, although SE seems to be safe, inexpensive and easy to perform in the evaluation of patients with high risk to develop stone recurrence, the compliance of the patients may be the restrictive parameter for the success.
\end{abstract}

Volume 5 Issue I - 2017

\author{
Kose Mustafa Gokhan,' Tekin Mehmet \\ Ilteris, ${ }^{2}$ Ozkardes Hakan ${ }^{2}$ \\ 'Department of Urology, Ordu State Hospital,Turkey \\ ${ }^{2}$ Department of Urology, Baskent University School of Medicine, \\ Turkey
}

Correspondence: Mustafa Gokhan Kose, Department of Urology, Ordu State Hospital, Baskent University School of Medicine, Ankara;Turkey, Tel 00 903/22/229/2,

Email vensyou@gmail.com

Received: March 10, 2016 | Published: June 23, 2017
Abbreviations: SE, simple metabolic evaluation; SWL, shock wave lithotripsy; URS, ureterorenoscopic modalities; PNL, percutaneous nephrolithotomy; $\mathrm{Ca}$, calcium; $\mathrm{Na}$, sodium; $\mathrm{K}$, potassium; $\mathrm{Mg}$, magnesium; CIT, citrate; OX, oxalate; $\mathrm{HCU}$, hypocitraturia; $\mathrm{HCaU}$, hypercalciuria; $\mathrm{CE}$, comprehensive evaluation; SF, stone formers

\section{Introduction}

Urolithiasis is one of the most common disorders in urology practice with an incidence of $0.4-1 \%$ in industrialized countries. ${ }^{1}$ Innovations in the therapeutic applications, such as extracorporeal shock wave lithotripsy (SWL), minimally invasive ureterorenoscopic modalities (URS) and percutaneous nephrolithotomy (PNL), have made most of the stones treatable. On the other hand, even for children, recurrence rates may reach to $50 \%$ in 5 to 10 years and $75 \%$ in 20 years without routine follow up and medical treatment. ${ }^{2-4}$ Patients carrying risk factors such as developmental urinary anomalies, genetic predisposition, excessive excretion of promoters or reduced excretion of inhibitors for crystallization are at high risk for new stone formation. ${ }^{5}$ These patients should be evaluated to reveal the underlying pathology for preventive medical therapy. ${ }^{6}$ Metabolic evaluation in patients with calculous disease reveals some form of abnormality in over $90 \%$ of patients. ${ }^{6}$ However, comprehensive metabolic evaluation is often neglected due to meticulous attention needed for urine collection, costs and dietary restriction. In this study, the efficacy of simple metabolic evaluation, commonly used for low risk stone formers, is evaluated in the management of high risk stone formers

\section{Materials and methods}

With the approval of Institutional Board and Local Ethic Committee (KA/0250), 53 patients (28 male, 25 female) of 268 patients (149 males, 119 females) regarded as high risk cases for stone recurrences were evaluated for 5 years, prospectively. These 53 patients attended routine controls in the 5 year period. The mean age of the patients was 46 (3-71) years. The patients underwent SWL, URS, PNL, open surgery or passed the stone spontaneously. One month later, all of the patients were checked for stone-free status by performing noncontrast spiral computerized tomography or intravenous urography. Stones were analysed by using X-ray diffractometry method. At the initial visit the patients were queried about the stone episodes, history of any surgery (gastrointestinal or genitourinary surgery), systemic diseases such as osteoporosis, sarcoidosis, etc., family history for urolithiasis, dietary methods and fluid intake, stone analysis, the age at the first episode and medication (Vit-D, calcium, etc).

All of the cases were informed about the risks and recurrence rates of urolithiasis. The patients taking any drugs liable to cause urinary calculi; who have a history of any bowel surgery, osteoporosis, sarcoidosis and first stone episode were included. Urine was analysed for $\mathrm{pH}$, glucose, density, microscopy for eryhtrocyte, leucocyte, crystallization (cystine) and culture. Calcium $(\mathrm{Ca})$, sodium $(\mathrm{Na})$, potassium $(\mathrm{K})$, magnesium $(\mathrm{Mg})$, creatinine, urate $(\mathrm{UA})$, parathormone in blood and $\mathrm{Ca}, \mathrm{Na}, \mathrm{K}, \mathrm{Mg}$, UA, citrate (CIT), urine volume and oxalate $(\mathrm{OX})$ in the sample of urine collected in 24 hours urination with standard diet were checked. The urine sample was collected in a dry and clean plastic container. No preservative material, such as hydrochloric acid, was used. The samples were delivered to the laboratory as soon as possible. In the laboratory urinary volume and $\mathrm{pH}$ were measured immediately, and then a part of it was acidified for urinary $\mathrm{OX}, \mathrm{Ca}, \mathrm{Na}, \mathrm{K}$ and CIT whereas the remaining was alkalinized for urinary UA calculation. The patients were treated according to the metabolic disorder determined by SE and were re-assessed in 6 months' period. The changes in blood and urine parameters after the treatment were examined. 


\section{Results}

The total numbers of the stones were $1.34 .^{1-3}$ Initial stone burdens were $28.5(10-300) \mathrm{mm}^{2}$. The stone analysis of the patients was as seen in Figure 1. In the beginning, 87\% (233/268) of the patients revealed metabolic abnormalities. Hypocitraturia (HCU) was detected in $60 \%$ of the patients as the most common patology (Table 1). The hypocitraturia was treated by potassium citrate of $30-60 \mathrm{mEq} /$ day and urine $\mathrm{pH}$ was evaluated in every visit. Despite the treatment, another pathology accompanied to HCU in $1(1 / 53)$ patient. In the end of the study, hypocitraturia was completely improved in $40 \%$ (21/53) of the patients. New stone formation was not reported (Table 2).

Hypercalciuria (HCaU) was established in $49 \%(26 / 53)$ of the subjects (Table 1). The hypercalciuric patients were treated by indapamide of 2.5 miligrams/day and potassium citrate $(30-60 \mathrm{mEq} /$ day). Potassium citrate was used to prevent the HCU secondary to indapamide treatment. During the follow up, stone recurrence occurred in only one patient in whom $\mathrm{HCaU}$ never improved. More than $50 \%$ of the patients with $\mathrm{HCaU}$ reached normal level by this treatment. Interestingly, when detected as the sole pathology, $\mathrm{HCaU}$ improved in all patients during the 5 years period (Table 2). Hyperoxaluria was determined in $32 \%$ (17/53) of the subjects (Table 1). It was treated by correcting the dietary habits and encouraging high fluid intake. It recovered in $41 \%$ of the patients. However, new stone formation appeared in 1 patient while hyperoxaluria persisted in 8 patients (Table 2 ).

During the five years, more than 3 parameters became abnormal in $9(17 \%)$ and less than three parameters became abnormal in 12 (23\%) patients. Interestingly, 5 of 7 patients whose parameters were normal initially, revealed various abnormalities during the follow up. In $5(1 \%)$ patients the same abnormal parameters never changed. In $14(26 \%)$ patients all of the parameters improved whereas at least one parameter recovered in $13(25 \%)$ patients (Table 2). Stone recurrence was seen in $2(4 \%)$ patients whose parameters were always abnormal. Primary hyperparathyroidism was detected in $5(9 \%)$ patients. These patients were treated by surgically. Urine volumes lower than $2000 \mathrm{ml}$ were accepted as a risk factor and were detected in $6(13 \%)$ patients. These cases were treated by adequate fluid intake to have a daily urine volume of $2000 \mathrm{ml}$. Urine volumes were turned to normal in 3 patients $(50 \%)$. New stone formation occurred in 1 patient Table $1 \& 2$.

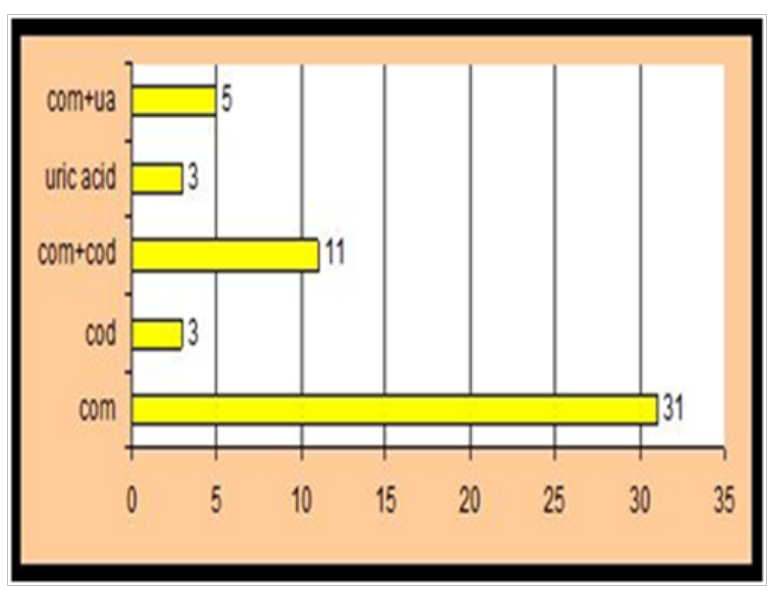

Figure I The types of the stones revealed by stone analysis. Com: calcium oxalate monohydrate Cod: calcium oxalate dehydrate

Ua: uric acide
Table I The results of simple metabolic evaluation in 53 patients who had rotine follow up for 5 years

\begin{tabular}{llll}
\hline Patology & Sole (\%) & Mixed (\%) & Total (\%) \\
\hline Hypercalciuria & $6(\mathrm{II})$ & $20(37)$ & $26(49)$ \\
Hyperoxaluria & 0 & $17(32)$ & $17(32)$ \\
Gout Diathesis & 0 & $2(4)$ & $2(4)$ \\
Hyperurisemia & 0 & $\mathrm{I}(2)$ & $\mathrm{I}(2)$ \\
Hyperuricosuria & 0 & $2(4)$ & $2(4)$ \\
Primary hyperparathyroidism & $\mathrm{I}(2)$ & $4(8)$ & $5(9)$ \\
Low urine volume & $2(4)$ & $4(8)$ & $6(13)$ \\
Other* & 0 & $\mathrm{I}(2)$ & $\mathrm{I}(2)$ \\
Normal*: Low urine ph, cystinuria, & $7(13)$ & 0 & $7(13)$ \\
etc. & & &
\end{tabular}

Table 2 Results after the treatment in 53 patients who had routine follow up for 5 years

\begin{tabular}{lllll}
\hline Parameter & \multicolumn{2}{l}{$\begin{array}{l}\text { Improved } \\
\text { (n) }\end{array}$} & $\begin{array}{l}\text { Impaired New } \\
\text { (n) }\end{array}$ & $\begin{array}{l}\text { No Charrence } \\
\text { (n) (n) }\end{array}$ \\
\hline Hypocitraturia & 13 & 1 & 8 & 10 \\
Hypercalciuria & 15 & 2 & 2 & 7 \\
Hyperoxaluria & 7 & 1 & 7 & 2 \\
Gout Diathesis & 2 & - & - & - \\
Hyperuricosuria & 2 & - & - & - \\
Hyperuricemia & - & - & - & 1 \\
Hyperparathyroidism & 3 & - & 2 & - \\
Low Urinary Volume & 3 & - & 1 & 2 \\
Other & 1 & - & - & - \\
Normal & - & 5 & - & 2 \\
\hline
\end{tabular}

\section{Discussion}

Nephrolithiasis is affecting 1-5\% of the population in industrialized countries. ${ }^{7}$ Most of these stones could be treated by SWL or endoscopic surgery (URS, PNL). However, high recurrence rates are still problem for either the patient or the clinician. ${ }^{4}$ Therefore, metabolic evaluation is a crucial step in the treatment of stone disease. Detailed history focusing on occupation, previous use of medications, dietary and lifestyle habits, recurrent urinary tract infections and underlying disorders that can predispose to stone formation should be examined. History and physical examination are the cornerstones in evaluation of stone formers. ${ }^{8}$ The patients should be classified as high (PHR) or low risk for recurrence (PLR). In the next step, SE or comprehensive evaluation (CE) are performed before preventive medical treatment.

Some authors stated that the stone recurrence rate was also important. Despite the high remission rates of medical prophylaxis in calcium stone formers (SF), medical prevention has been mentioned to be non cost effective in patients forming stones less frequently than once every 3 years. ${ }^{7,9,10}$ In the present study stone formation period less than one in 3 years were excluded. Simple metabolic evaluation is recommended in patients with low risk for stone recurrence.

However, it is debate whether a single 24- hour urine collection is enough for metabolic evaluation, or not. Some authors stated that only one urine specimen was not enough in metabolic evaluation to reflect the pathology. ${ }^{11,12}$ One should be collected under random diet and the other after restriction. This may be useful especially for classifying the subtypes of hyper calciuria. On the other hand, Pak et al supports a single 24 hour urine collection. ${ }^{13}$ In the study, we checked the sample of urine collected in 24 hours urination with standard diet. However, distinct from the other studies, we evaluated patients with high risk for stone recurrence. Preservation of urine seemed to be difficult by the method performed in the study, since we had only one urine sample 
to investigate. Ferraz et al concluded that post delivery acidification or alkalinization of urine samples did not modify the measured levels of urinary $\mathrm{OX}, \mathrm{Ca}, \mathrm{Mg}$, creatinine and UA and change on CIT was not relevant allowing all parameters to be determined in a single urine sample [14]. We measured urinary volume, $\mathrm{pH}$ in the 24 hour urine sample, and the half part of the sample was acidified for $\mathrm{OX}, \mathrm{Ca}, \mathrm{Mg}$ and CIT calculation and the other half part was alkalanized for UA determination as Ferraz et al recommended. ${ }^{14}$

During 5 years, approximately $80 \%$ of the patients who did not continue their visits were excluded from the study. When these patients were called up for visits, they refused since most of them considered the process as hopeless, time consuming, bothersome and expensive. They were acquainted about stone recurrence rates, complications and importance of metabolic evaluation in stone disease again, only $10 \%$ accepted to continue their routine follow ups. This point out the importance of patient compliance. In our opinion, the compliance of the patient is the most essential factor and it might be less than $80 \%$ if the patient had to collect more than one 24 hours urine sample. Also, dietary restriction would help to reduce the participation.

In a recent study it is stated that $\mathrm{CE}$ is superior to SE (a single 24 hour urine collection) neither in cost nor in efficacy. ${ }^{15}$ We did not investigate the cost effectiveness, but Lotan et al..$^{15}$ estimated that only small differences between empiric and conservative therapy were demonstrated in recurrent stone formers in countries such as Turkey and Switzerland. This suggestion is very important when informing the patients about the cost effectivity. It is stated that with appropriate evaluation, a cause for stone formation can be found in $97 \%$ of the patients. ${ }^{16}$ We determined abnormality in almost $90 \%$ of the patients by SE. This is close to the rates determined by CE. When it is thought that 215 patients were out of follow up in the present study, it may be suggested that this rate might be much more than it has been estimated. Stone analysis is warranted in all patients and may provide guidance for the therapeutic treatment. Kourambas et al. ${ }^{17}$ stated that non calcareous stone forming patients reflected specific metabolic disorders. The authors suggested that compositional stone analysis should be an integral part of the metabolic evaluation of patients with nephrolithiasis and stone analysis alone may provide guidance for therapeutic treatment and obviate a formal metabolic evaluation. In our study the patients whose stone analysis could not be detected, were excluded.

Metabolic evaluation and preventive medical therapy are supplementary steps in the treatment of urolithiasis and the patient's compliance is an essential part of the process. Even in the first visit SE may enlighten the pathology as high as $87 \%$. However, the participation may be as low as $25 \%$ during the follow up. Why do the patients get out of the follow up? The main reason might be the opinion to have undergone to an evaluation that is expensive, time consuming, bothersome and useless. Before treatment, all of the patients must be informed about their disease, risks and the necessity of the evaluation. We conclude that, although SE seems to be safe, inexpensive and easy to perform in the evaluation of PHR, the patients' compliance may be the restrictive parameter for the success. However, SE may be very useful for the clinician to determine the pathology even in the first visit.

\section{Acknowledgments}

None.

\section{Conflicts of Interset}

None.

\section{References}

1. Lotan Y, Cadeddu JA, Pearle MS. International comparison of cost effectiveness of medical management strategies for nephrolithiasis. Urol Res. 2005;33(3):223-230.

2. Cohen TD, Ehreth J, King LR, et al. Pediatric urolithiasis: Medical and surgical management. Urology. 1996;47(3):292-305.

3. Sutherland J, Parks JH, Coe F. Recurrence after a single renal stone in a community practice. Miner Electrolyte Metab. 1985;11(4):267-269.

4. Trinchieri A, Ostini F, Nespoli R, et al. A prospective study of recurrence rate and risk factors for recurrence after a first renal stone. $J$ Urol. 1999;162(1):27-30.

5. Heilberg IP, Schor N. Renal stone disease: Causes, evaluation and medical treatment. Arq Bras Endocrinol Metabol. 2006;50(4):823-831.

6. Pak CY, Britton F, Peterson R, et al. Ambulatory evaluation of nephrolithiasis. Classification,clinical presentation and diagnostic criteria. Am J Med. 1980;69(1):19-30.

7. Delvecchio FC, Preminger GM. Medical management of stone disease. Curr Opin Urol. 2003;13(3):229-233.

8. Heilberg IP, Schor N. Renal Stone Disease: Causes, Evaluation and Medical Treatment. Arq BrasEndocrinol Metab. 2006;50(4):823-831.

9. Chandhoke PS. When is medical prophylaxis cost-effective for recurrent calcium stones? J Urol. 2002;168(3):937-940.

10. Pearle MS, Roehrborn CG, Pak CY. Meta-analysis of randomized trials for medical prevention of calcium oxalate nephrolithiasis. J Endourol. 1999;13(9):679-685.

11. Parks JH, Goldfisher E, Asplin JR, et al. A single 24-hour urine collection is inadequate for the medical evaluation of nephrolithiasis. $J$ Urol. 2002;167(4):1607-1612

12. Yagisawa T, Chandhoke PS, Fan J. Comparison of comprehensive and limited metabolic evaluations in the treatment of patients with recurrent calcium urolithiasis. J Urol. 1999;161(5):1449-1452.

13. Pak CY, Peterson R, Poindexter JR. Adequacy of a single stone risk analysis in the medical evaluation of urolithiasis. $J$ Urol. 2001;165(2):378-381.

14. Ferraz RR, Baxmann AC, Ferreira LG, et al. Preservation of urine samples for metabolic evaluation of stone-forming patients. Urol Res. 2006;34(5):329-337.

15. Lotan Y, Cadeddu JA, Roerhborn CG, et al. Cost-effectiveness of medical management strategies for nephrolithiasis. J Urol. 2004;172(6 Pt 1):2275-2281.

16. Pak CY. Southwestern Internal Medicine Conference: medical management of nephrolithiasis: A new simplified approach for general practice. Am J Med Sci. 1997;313(4):215-219.

17. Kourambas J, Aslan P, Teh CL, et al. Role of stone analysis in metabolic evaluation and medical treatment of nephrolithiasis. $J$ Endourol. 2001;15(2):181-186. 